\title{
First Principles Investigations on the Electronic and Magnetic Properties of $\mathrm{La}_{4} \mathrm{Ba}_{2} \mathrm{Cu}_{2} \mathrm{O}_{10}$
}

\author{
Shalika Ram Bhandari ${ }^{1,2}$, Ram Kumar Thapa ${ }^{1,3}$, and Madhav Prasad Ghimire ${ }^{1^{*}}$ \\ ${ }^{1}$ Condensed Matter Physics Research Center, Butwal, Rupandehi, Nepal \\ ${ }^{2}$ Department of Physics, Bhairahawa Multiple Campus, Tribhuwan University, Rupandehi, Nepal \\ ${ }^{3}$ Department of Physics, Mizoram University, Aizawl 796009, Mizoram, India \\ *Correspondence to: ghimire.mpg@gmail.com
}

\begin{abstract}
Electronic and magnetic properties of $\mathrm{La}_{4} \mathrm{Ba}_{2} \mathrm{Cu}_{2} \mathrm{O}_{10}$ had been studied by first-principles density functional theory (DFT). Based on the DFT calculation $\mathrm{La}_{4} \mathrm{Ba}_{2} \mathrm{Cu}_{2} \mathrm{O}_{10}$ is found to have a ferromagnetic (FM) ground state. The material undergo charge-transfer type insulator to Mott-Hubbard type insulator transition which happens due to strong correlation in La-4f and $\mathrm{Cu}$-3d states. Our results show that the $3 d$ electrons of $\mathrm{Cu}$ hybridize strongly with $O-2 p$ states near the Fermi level giving rise to the insulating state of $\mathrm{La}_{4} \mathrm{Ba}_{2} \mathrm{Cu}_{2} \mathrm{O}_{10}$. Our study suggests that the enhanced magnetic moment is a result of itinerant exchange rather than the exchange interaction involving individual ions of $\mathrm{Cu}$ atoms. The total magnetic moment calculated in the present studies is $2 \mu_{B}$ per unit cell for $\mathrm{La}_{4} \mathrm{Ba}_{2} \mathrm{Cu}_{2} \mathrm{O}_{10}$.
\end{abstract}

Keywords: Density functional theory, lanthanide cuprates, electron-correlation effects, ferromagnetic Mottinsulator.

\section{Introduction}

The discovery of superconductivity in ceramic materials has been a strong race to understand the mechanism of superconductivity in $\mathrm{CuO}$ materials, in particular how magnetism influences its behavior. It has been found that at very low doping, cuprates show the long range order of antiferromagnet, doping breaks up the antiferromagnetic order and becomes an insulator. Only with doping fraction between about 0.1 and 0.2 , they become superconductors [Bednortz and Muller, 1986; Freeman and Xu, 1987]. The understanding which has emerged is that copper oxide layers provide the path for currents. These copper oxide layers are separated by layers of other atoms which serve as spacers and charge reservoirs [Matthias,
1954; Mizuno et al., 1990]. Since the discovery of high temperature superconductors, many new materials have been invented. Precise physical and chemical work on high temperature superconductors has made tremendous progress in the theoretical and experimental study of physical properties and carrier state characterizations [Yahua et al., 2015; Ghimire et al., 2011, 2015; Rai et al., 2015]. Since the discovery of first transition metal oxide has obvious benefits of room temperature superconductors, which conduct electricity without resistance, may include lossless power generator, transformers and transmission lines, powerful supercomputers and even superfast magnetically levitated trains. Needless to say the discovery of room temperature superconductors 
would revolutionize the way we use and generate power. Cuprates are the king of super conductors [Amin et al., 2011; Wannier, 1937]. Although no theory on the mechanism of high temperature superconductivity has gained acceptance, the observation of high temperature superconductivity in this class of layered materials has led to a phenomenological understanding that superconductivity depends on the two dimensional conducting planes with weak interplane coupling. The compound $\mathrm{Ln}_{4} \mathrm{Ba}_{2} \mathrm{Cu}_{2} \mathrm{O}_{10}$,where $\mathrm{Ln}=$ Lanthanide) is a ceramic material which is a new class lanthanide high temperature superconductors obtained by substituting barium atoms into the lattice of lanthanum copper oxide in a solid solution [Paukov,1991; Good Enough, 1958].

The overall feature of the specific heat in $\mathrm{La}_{4} \mathrm{Ba}_{2} \mathrm{Cu}_{2} \mathrm{O}_{10}$ agrees to that of the mean field approximation with $1 / 2$ spin system and is more 3-dimensional than that of 2-dimensional $\mathrm{Cu}$ ferromagnet $\mathrm{K}_{2} \mathrm{CuF}_{4}$. The measured magnetizations on the single crystals and the estimated anisotropy suggest the Heisenberg system with Ising-like anisotropy due to the comparably large interlayer coupling. In the system of $\mathrm{Nd}$ substitution for $\mathrm{La}$, the ferromagnetic transition is not observed because $\mathrm{Nd}$ ions having the $f$-spins may disturb the exchange interaction between interlayer $\mathrm{Cu}^{2+}$ ions. The study of magnetic properties of $\left(\mathrm{La}_{1-\mathrm{x}} \mathrm{R}_{\mathrm{x}}\right)_{4} \mathrm{Ba}_{2} \mathrm{Cu}_{2} \mathrm{O}_{10}$ for $\mathrm{R}=\mathrm{Nd}, \mathrm{Sm}, \mathrm{Gd}$ and $\mathrm{Eu}$, of which the end material $\mathrm{La}_{4} \mathrm{Ba}_{2} \mathrm{Cu}_{2} \mathrm{O}_{10}$ is known as a rather exceptional ferromagnet among the known copper-oxide insulators. It was found that the average ionic radius $r$ of the elements at the La site determines the solubility limit of $\mathrm{R}$ elements. When $r$ is larger than $\sim 1.11$
$\AA$, the compound has the $\mathrm{La}_{4} \mathrm{Ba}_{2} \mathrm{Cu}_{2} \mathrm{O}_{10}$ type structure, while for smaller $r$, the $\mathrm{Y}_{2} \mathrm{BaCuO}_{5}$ type structure is formed [Vaughey et al., 1991; Khomskii and Kugel, 1973; Perdew et al., 1996]. The magnetic behavior of the compounds depended on the $\mathrm{R}$ element, even a rather small amount of $\mathrm{Nd}$ altered the ferromagnetic ground state to an antiferromagnetic one, with a possible spin-glass state in between [Jongh and Miedema,1974; Ghimire et. al. 2011; 2015; Rai et al, 2015]. For $\mathrm{R}=\mathrm{Sm}$ and $\mathrm{Gd}$, the experimental results suggest a similar change of ferromagnetism to antiferromagnetism, but a ferrimagnet [Ghimire et al., 2015] like behavior was observed for intermediate compositions. On the other hand, ferromagnetism resided up to the solubility limit for $\mathrm{R}=\mathrm{Eu}$. The application of high pressure upto $8.0 \mathrm{GPa}$ hardly affected the magnetism of $\mathrm{La}_{4} \mathrm{Ba}_{2} \mathrm{Cu}_{2} \mathrm{O}_{10}$. This result, as well as the results of Rietveld analysis of R-substituted $\left(\mathrm{La}_{1-\mathrm{x}} \mathrm{R}_{\mathrm{x}}\right)_{4} \mathrm{Ba}_{2} \mathrm{Cu}_{2} \mathrm{O}_{10}$, indicate that the reduction of ferromagnetism with the increase in $\mathrm{x}$ is not attributable to the structural change induced by the substitution, the chemical pressure effect.

Few investigation were done theoritically to study the properties of $\mathrm{La}_{4} \mathrm{Ba}_{2} \mathrm{Cu}_{2} \mathrm{O}_{10}$ using Hubbard model by Mizuno et al., they observed that most of the half-filled cuprates shows antiferromagnetic property in ground state while some of the cuprates possesses ferromagnetic behavior [Meinert, 2013]. Similarly the isostructural $\mathrm{Nd}_{4} \mathrm{Ba}_{2} \mathrm{Cu}_{2} \mathrm{O}_{10}$ are found to allign antiferromagnetically below $7.5 \mathrm{~K}$ but the halffilled $\mathrm{Cu}$-d orbitals in $\mathrm{La}_{4} \mathrm{Ba}_{2} \mathrm{Cu}_{2} \mathrm{O}_{10}$ are found to align ferromagnetically below $5 \mathrm{~K}$ [Wei et. al., 2002]. This type of unusual insulating FM phase possesses a great challenge to our quantitative 
theoretical understanding of microscopic mechanism involved in real materials. There are few attempts done for quantitative processes to study the intriguing behavior of $\mathrm{La}_{4} \mathrm{Ba}_{2} \mathrm{Cu}_{2} \mathrm{O}_{10}$. The description of Hubbard like model is found to lack the identity of destructive interference of hoping paths which suppress AF coupling and give a small FM coupling along $\mathrm{z}$ axis in a narrow parameter range as assumed by Good enough et al. [Marzari and Vanderbilt, 1997]. Furthermore the assumption of FM coupling between the nearest neighbors in the plane is apparently in contradiction to the AF order in $\mathrm{Nd}_{4} \mathrm{Ba}_{2} \mathrm{Cu}_{2} \mathrm{O}_{10}$ [Subramanian, 1988].

In order to resolve this problem theoretically quantum magnetism model was introduced with adjustable parameters. Though this approach is intuitive and computationally manageable but this lack to describe the quantitative detail of the complex interaction that occurs in real materials and also lack to describe the spin spin coupling in magnetic orbit. A very good attempt was made by [Wei et al., 2002 and Ford, 2005] to resolve the problem of microscopic mechanism of the unusual insulating ferromagnetism in $\mathrm{La}_{4} \mathrm{Ba}_{2} \mathrm{Cu}_{2} \mathrm{O}_{10}$. They experimented that the dominant mechanism turns out to be intersite FM direct exchange that is currently ignored in the macroscopic studies of this system [Smirnov and Usvyat, 2001]. This process occurring mainly at the $\mathrm{La}$ and $\mathrm{O}$ sites overwhelms the weak tendency towards AF order via Hubbard type super exchange [Jonker and Van Santen, 1950; Wu et al.,1987; Sun and In-Hwan $O h, 1996]$. On the other hand, on $\mathrm{Nd}_{4} \mathrm{Ba}_{2} \mathrm{Cu}_{2} \mathrm{O}_{10}$ crucial dominant direct exchange unlike the nearest neighbor as assumed by Hubbard but with sites above or below them. This generates the proper in plane order. Finally, the role of the chemical effect is introduced with numerical simulation of $\mathrm{La}_{4} \mathrm{Ba}_{2} \mathrm{Cu}_{2} \mathrm{O}_{10}$ under pressure which suggests an intriguing pressure induced FM to AF transition [Castano et al., 2003; Slater, 1960].

\section{Crystal Structure and Computational Details}

The compound $\mathrm{La}_{4} \mathrm{Ba}_{2} \mathrm{Cu}_{2} \mathrm{O}_{10}$ is tetragonal with space group $P_{4} / \mathrm{mbm}$. In this compound ten and eight coordinate cation sites $(2 \mathrm{~b}$ and $4 \mathrm{~g}$ ) are selectively occupied by $\mathrm{Ba}^{2+}$ and $\mathrm{Ln}^{3+}$ respectively, and no oxygen deficiency is observed in this compound [Subramanian, 1988]. The compound consists of five independent and eighteen total atoms in a unit cell.

The electronic and magnetic properties of $\mathrm{La}_{4} \mathrm{Ba}_{2} \mathrm{Cu}_{2} \mathrm{O}_{10}$ are studied by using full-potential linearized augmented plane wave (FP-LAPW) method based on density functional theory (DFT) as implemented in the WIEN2k code [Blaha, 2001]. The core states are treated fully relativistically while the semi-core and valence states are treated semi-relativistically. The standard generalized-gradient approximation (GGA) exchange correlation potential within the PBE-scheme [Anisimov et al., 1997] were used with Coulomb interaction $\mathrm{U}$ due to strong electron-electron interaction in $\mathrm{La}$ and $\mathrm{Cu}$ ions. The results shown here is with $\mathrm{U}_{\mathrm{La}}=6 \mathrm{eV}$ and $\mathrm{U}_{\mathrm{Cu}}=5 \mathrm{eV}$ respectively. We have chosen the muffin-tin (MT) radii for $\mathrm{La}, \mathrm{Ba}, \mathrm{Cu}$, and $\mathrm{O}$ to be $2.34,1.92,1.99$ and 1.77 a.u. respectively. Integrations in reciprocal space were performed 


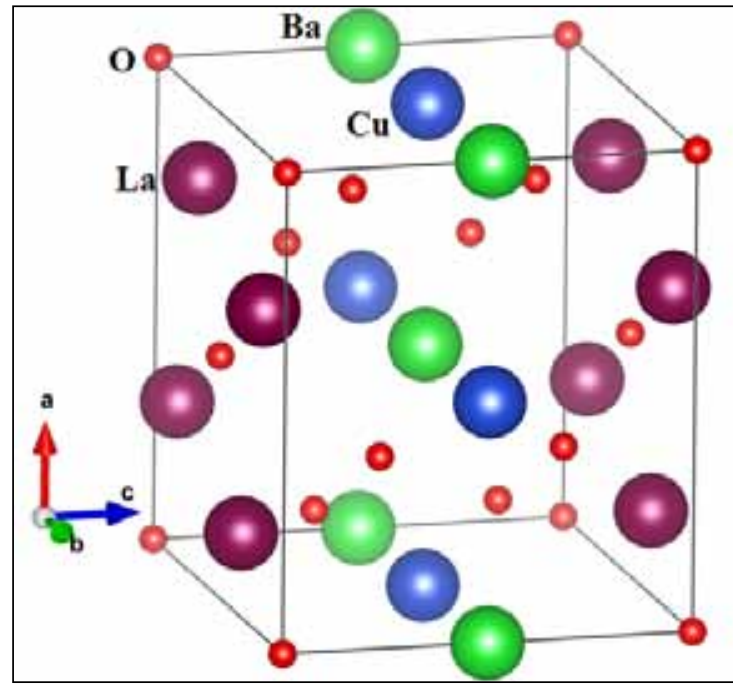

Figure 1. Crystal structure of $\mathrm{La}_{4} \mathrm{Ba}_{2} \mathrm{Cu}_{2} \mathrm{O}_{10}$ (oxygen atoms are co-ordinated to $\mathrm{Cu}$-atoms).

using 108 spatial $k$-points in the irreducible wedge of the Brillouin zone.

\section{Results and Discussions}

$\mathrm{La}_{4} \mathrm{Ba}_{2} \mathrm{Cu}_{2} \mathrm{O}_{10}$ belongs to tetragonal cuprates family where La at A-site provides charge to the system and nominally take the charge state +3 with $4 \mathrm{f}^{0}$ configuration, lying in the conduction region above the Fermi level $\left(E_{\mathrm{F}}\right)$. The transition element $\mathrm{Cu}$ nominally takes the charge state +2 with $3 \mathrm{~d}^{9}$ configuration where nine out of ten $d$ orbits are occupied lying in the valence region, while the remaining un-occupied state lie in the conduction region due to large crystal field from oxygen ions.

For the ground state investigations of $\mathrm{La}_{4} \mathrm{Ba}_{2} \mathrm{Cu}_{2} \mathrm{O}_{10}$ we first consider the nonmagnetic calculations with the standard GGA method. This provides a reference for studying the magnetic phases. From the partial density of states (DOS) for non-magnetic phase as shown in Fig. 2, the $\mathrm{Cu}$-d states lying at the top of the valence band has a sharp peak indicating localized states which is an indication of the unstable ground state. As observed from Fig. 1, Ba states has no impact on the electronic states as they lie deep in the core region whereas the $\mathrm{Cu}-3 \mathrm{~d}$ and $\mathrm{O}-2 \mathrm{p}$ states hybridize strongly in the valence region indicative of the possibility of magnetic ground state. La-4f states on
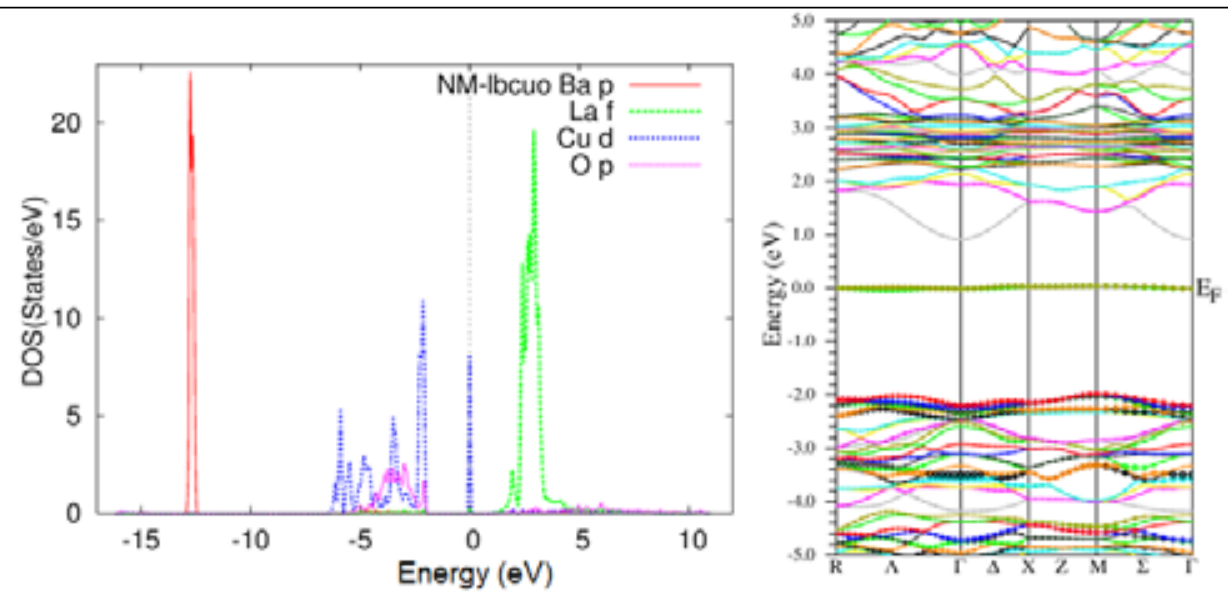

Figure 2. Partial density of states (DOS) and band structure for non-magnetic phase of $\mathrm{La}_{4} \mathrm{Ba}_{2} \mathrm{Cu}_{2} \mathrm{O}_{10}$ within GGA 
the other hand lies in the conduction region with no contribution to the electronic states. Furthermore, the material $\mathrm{La}_{4} \mathrm{Ba}_{2} \mathrm{Cu}_{2} \mathrm{O}_{10}$ is found to be a charge-transfer type insulator.

In order to know the experimental ground state we calculated the total energies for nonmagnetic (NM), ferromagnetic (FM) and different phase of antiferromagnetic (AFM) configurations. Our calculation shows that FM configuration is the stable ground state consistent with the experimental prediction with total energy of $\sim 1.5 \mathrm{eV}$ less than the NM and $\sim 10 \mathrm{meV}$ less than the AFM's configurations.

To understand the electronic properties of $\mathrm{La}_{4} \mathrm{Ba}_{2} \mathrm{Cu}_{2} \mathrm{O}_{10}$ we carry out the DOS and band structure calculations for the FM phase as shown in Fig. 3 and Fig. 4. Since $\mathrm{La}$ and $\mathrm{Cu}$ are strongly correlated, GGA+U scheme was considered. According to our first-principles DFT calculations, the material $\mathrm{La}_{4} \mathrm{Ba}_{2} \mathrm{Cu}_{2} \mathrm{O}_{10}$ is found to be Mott insulator (see Fig. 3) with an energy gap of approx. $2.1 \mathrm{eV}$ at the Fermi level $\left(E_{\mathrm{F}}\right)$. The gap is formed between the $\mathrm{Cu}-\mathrm{d}$ and La-4f states in spin-up while that between $\mathrm{Cu}-\mathrm{d}$ states in spin-down channels.

From the total and partial $(\mathrm{La}-4 \mathrm{f}, \mathrm{Cu}-$ 5d, O-2p) DOS shown in Fig. 3 and the band structure in Fig. 4 for spin-up and spin-down channels, it is observed that the empty La- $4 f$ states lies above $E_{\mathrm{F}}$ for both spin-channels in the conduction region. The $\mathrm{Cu}-3 d$ states are found to play key role in dictating the electronic properties of $\mathrm{La}_{4} \mathrm{Ba}_{2} \mathrm{Cu}_{2} \mathrm{O}_{10}$. They are found to hybridize strongly with the $\mathrm{O}-2 \mathrm{p}$ states in both spin channels. Their hybridization occurs mostly in the valence region near $E_{\mathrm{F}}$ and in the conduction region. We observe that $\mathrm{Cu}-\mathrm{d}$ (i. e., especially $d_{e g}$ ) states are fully occupied in spinup channels and thus lies in the valence region whereas in spin-down channel, only one out of two $d_{e g}$ states are occupied while the remaining states being empty lies in the conduction region hybridizing with $\mathrm{O}-2 \mathrm{p}$ and La- $4 \mathrm{f}$ states. Charge transfer effect is prominent between $\mathrm{Cu}-3 \mathrm{~d}$ and $\mathrm{O}-2 \mathrm{p}$ states due to strong hybridization. We observe that there is large exchange splitting

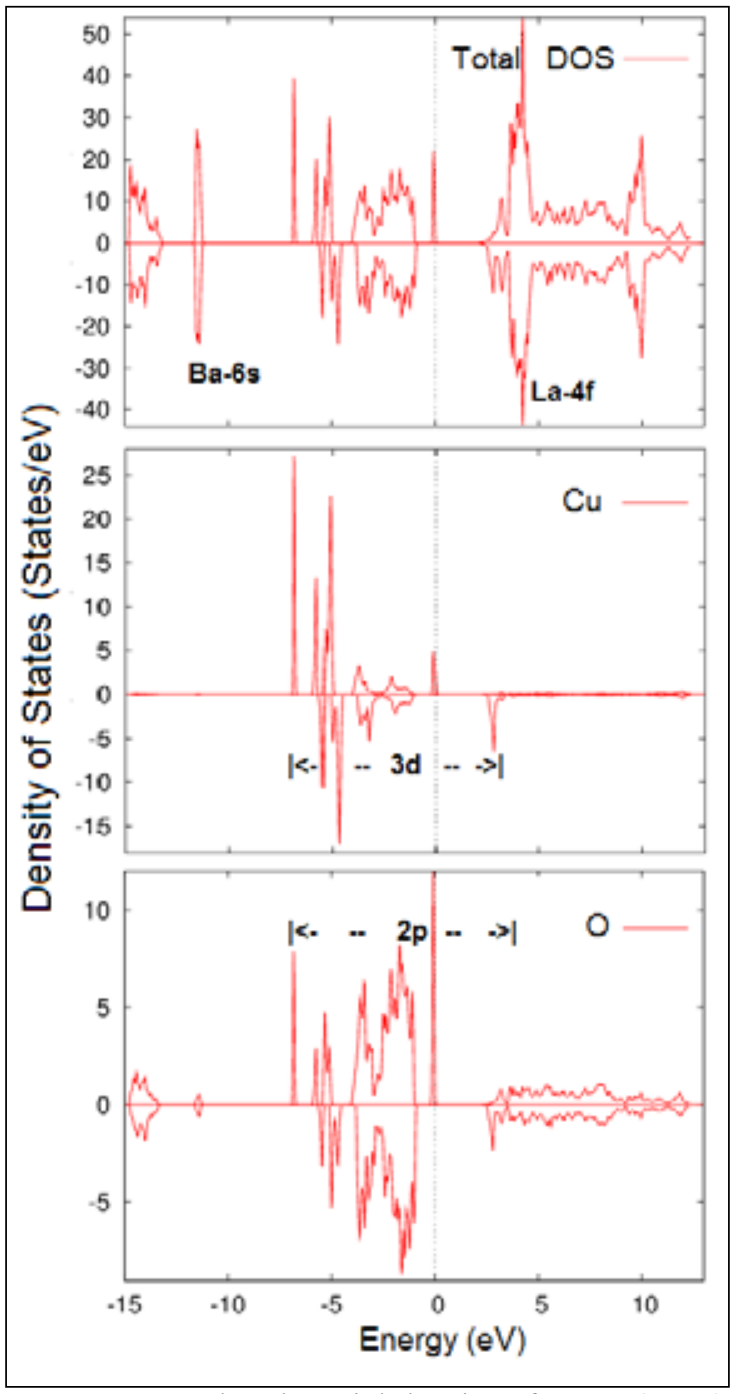

Figure 3. Total and partial density of states (DOS) of $\mathrm{La}_{4} \mathrm{Ba}_{2} \mathrm{Cu}_{2} \mathrm{O}_{10}$ for the ferromagnetic ground state. 


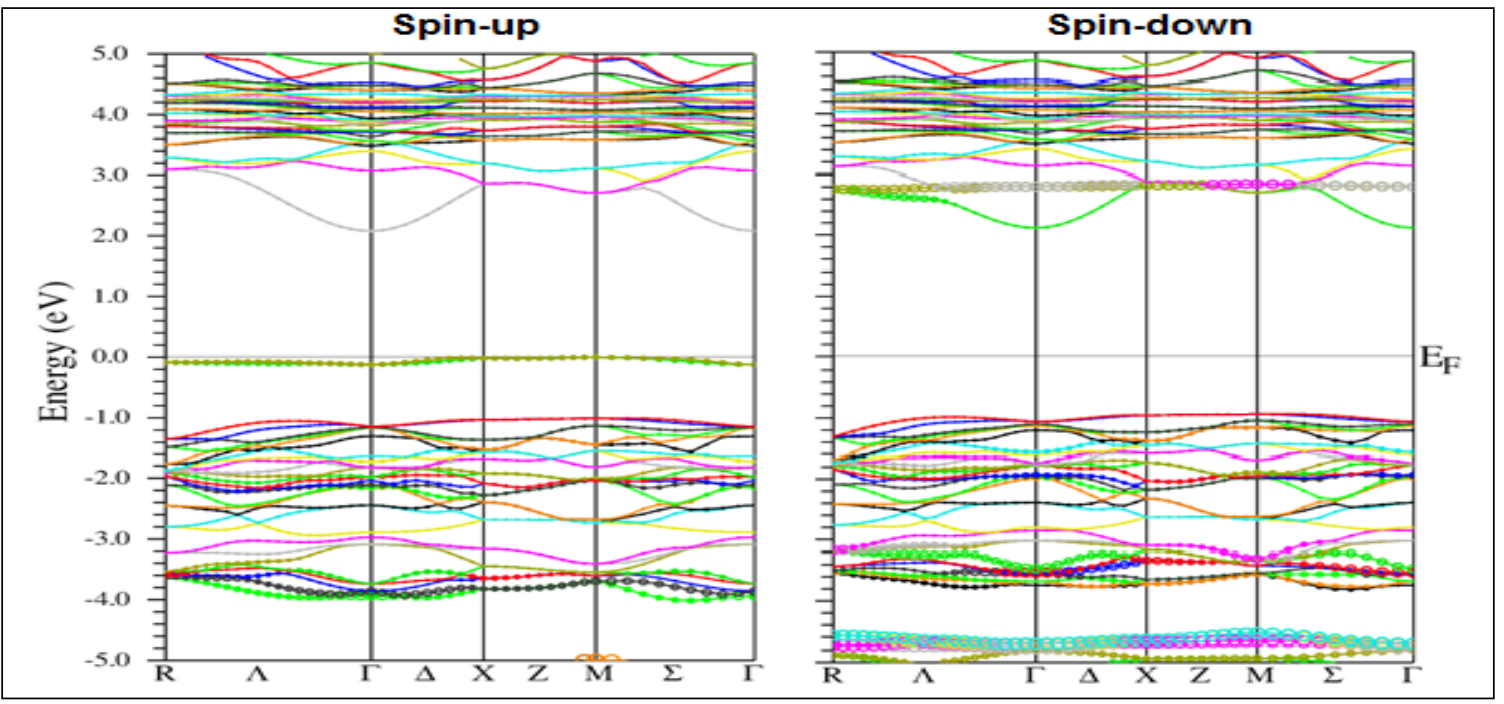

Figure 4. Band structures of $\mathrm{La}_{4} \mathrm{Ba}_{2} \mathrm{Cu}_{2} \mathrm{O}_{10}$ for spin-up (left) and spin-down (right) channels.

between $\mathrm{Cu}-3 \mathrm{~d}$ states between the spin-up and spin-down states whereas for La-4f there is no exchange splitting due to vacant orbitals. Exchange splitting energy of $\mathrm{Cu}-3 \mathrm{~d}$ is found to be $\sim 4 \mathrm{eV}$ which contributes to ferromagnetic behavior in $\mathrm{La}_{4} \mathrm{Ba}_{2} \mathrm{Cu}_{2} \mathrm{O}_{10}$.

From the band structure calculations shown in Fig. 4, the fat band appearing suggest the contributions from $\mathrm{Cu}-3 \mathrm{~d}$ states while the contributions to conduction region are dominantly from the La-4f states with partial contributions from $\mathrm{Cu}$-d states in spin-down channel. As observed clearly, $\mathrm{La}_{4} \mathrm{Ba}_{2} \mathrm{Cu}_{2} \mathrm{O}_{10}$ $\mathrm{Cu}-3 \mathrm{~d}$ states fully occupy the valence region for spin-up as well as spin-down channels. The band structure plot depicts $\mathrm{La}_{4} \mathrm{Ba}_{2} \mathrm{Cu}_{2} \mathrm{O}_{10}$ to be an indirect band Mott-Hubbard type insulator with a band gap of $2.1 \mathrm{eV}$. From first-principles calculations, we obtain the magnetic moment of $\mu \approx 0.7 \mu_{\mathrm{B}}$ per $\mathrm{Cu}$ atom, and $\mu \approx 0.1 \mu_{\mathrm{B}}$ per $\mathrm{O}$ atoms, respectively, with a total magnetic moment of $\mu_{\text {tot }}=2.0 \mu_{\mathrm{B}}$ per formula unit in La${ }_{4} \mathrm{Ba}_{2} \mathrm{Cu}_{2} \mathrm{O}_{10}$.

\section{Conclusions}

Based on the first-principles density functional calculations, we predict $\mathrm{La}_{4} \mathrm{Ba}_{2} \mathrm{Cu}_{2} \mathrm{O}_{10}$ to be a ferromagnetic insulator consistent with the experiment. Our study suggests that the magnetic moment is a result of itinerant exchange and the exchange interaction involving individual $\mathrm{Cu}$ ions.

\section{Acknowledgments}

This work was supported partially by CMPRC, Butwal (Nepal) and the University Grants Commision, Nepal. Editor N. P. Chapagain thanks the reviewers for their assistance in evaluating this paper.

\section{References}

Amin, B., Ahmad, I., Magboal, M., Gourrisai, S. and Ahmad, R. (2011), On the Non-Orthogonality Problem Connected with the Use of Atomic Wave Functions in the Theory of Molecules and Crystals. J. App. Phys. 109, 023-109.

Anisimov,V. I., Aryasetiawan F. and Lichtenstein A. I. (1997), J. Phys.: Condens. Matter 9, 767-808. 
Bednortz, J.G. and Muller, K.A. (1986), Possible high Tc supsuperconductivity in La- $\mathrm{Ba}-\mathrm{Cu}-\mathrm{O}$. Phys. Rev. B 64, 189-193.

Blaha, P., Schwarz, K., Madsen, G. K. H., vasnicka, D. K. and Luitz, J., (2001), WIEN2k. An Augmented Plane Wave and Local orbitals Program for Calculating Crystal Properties. Technische Universität Wien, Vienna.

Castano, O., Cavallaro, A., Palau, A., Gonzalez, J. C., Rossell, M., Puig T., Sandiumenge F., Mestres, N., Pinol, S., Pomar, A., and Obradors, X. (2003), High quality $\mathrm{YBa}_{2} \mathrm{Cu}_{3} \mathrm{O}_{\{7-\mathrm{x}\}}$ thin films grown by trifluoroacetates metal-organic deposition. Supercond. Sci. Technol.16, 45-53.

Ford, P.J., and Saunders, G. A. (2005). The rise of the superconductors. First Edition CRC Press, Florida. ISBN-07484-0772-3.

Ghimire, M. P., Thapa, R. K., Rai, D. P., Sandeep, Sinha, T. P, and Hu, X. (2015), Half metallic ferromagnetism in tri-layered perovskites $\mathrm{Sr}_{4} \mathrm{~T}_{3} \mathrm{O}_{10}(\mathrm{~T}=\mathrm{Co}, \mathrm{Rh})$. J. Appl. Phys. 117, 063903.

Ghimire, M. P., Wu, L. H., and $\mathrm{Hu}, \mathrm{X}$. (2015), Half metallic ferrimagnetism in hole-doped lanthanide iridates. J. Supercond. Nov. Magn. 28, 917-919.

Ghimire, M. P., Sandeep, Sinha, T. P., and Thapa, R. K. (2011), First principles study of the electronic and magnetic properties of semi-Heusler alloys $\mathrm{NiXSb}(\mathrm{X}=\mathrm{Ti}, \mathrm{V}, \mathrm{Cr}$ and $\mathrm{Mn})$. J. Alloys Compds. 509, 9742-9752.

Ghimire, M. P., Sandeep, Sinha, T. P, and Thapa, R. K. (2011), Ground state electronic and magnetic properties of $\mathrm{RCrSb}_{3}(\mathrm{R}=\mathrm{La}, \mathrm{Ce}, \mathrm{Sm}, \mathrm{Gd}, \mathrm{Dy})$ : A first principles study. Sol. Stat. Commn. 151, 1224-1227.

Ghimire, M.P., Sandeep, Sinha, T. P. and Thapa, R.K. (2011), First principles study of the electronic and optical properties of $\mathrm{SbTaO}_{4}$, Physica B: Condensed Matter 406, 3454-3457.
Good Enough, J.B. (1958), An interpretation of the magnetic properties of the perovskite-type mixed crystals $\mathrm{La}_{1-\mathrm{x}} \mathrm{Sr}_{\mathrm{x}} \mathrm{CoO}_{3-\lambda}$. J. Phys. Chem Solids 6, 287-297.

Jongh, L. J. D. and Miedema, A.R. (1974), Experiments on simple magnetic model systems. Adv. Phys. 23, 1-5.

Jonker, G.H. and Van Santen, J. H. (1950), Ferromagnetic compounds of manganese with perovskite structure. Physica 16, 337-349.

Kook, S.Y and Oh, I.H. (1996), Preparation of Ultrafine $\mathrm{YBa}_{2} \mathrm{Cu}_{3} \mathrm{O}_{7-\mathrm{x}}$ Superconductor Powders by the Poly (vinyl alcohol)-Assisted Sol-Gel Method. Ind. Eng. Chem. Res. 35 (11), 42965114.

Mizuno, F. Masuda, H, Hirabayashi, I, Tanaka, S. Hasegawa, M. and Mizutani, U. (1990), Lowtemperature ferromagnetism in $\mathrm{La}_{4} \mathrm{Ba}_{2} \mathrm{Cu}_{2} \mathrm{O}_{10}$. Nature 345, 788-789.

Matthias, B.T. (1954), Superconductivity of $\mathrm{Nb}_{3} \mathrm{Sn}$. Phys. Rev. 95, 1435-1437.

Marzari, N. and Vanderbilt N. (1997), Maximally localized generalized Wannier functions for composite energy bands. Phy. Rev. B 56, 12847 12848

Meinert, M. (2013), Electronic structure and optical band gap determination of $\mathrm{NiFe}_{2} \mathrm{O}_{4}$. Phys. Rev. $B$ 87, $045103-045104$.

Paukov, I.V. (1991), Magnetic phase transition and short range order in $\mathrm{Nd}_{2} \mathrm{BaCuO}_{5}$. Phys. Lett. A 157, 306-315.

Perdew, J. P., Burke K. and Ernzerhof, M. (1996), Generalized-Gradient Approximations made simple. Phys. Rev. Lett. 77, 3865-3996.

Rai, D. P., Shankar, A., Ghimire, M. P., Sandeep, and Thapa, R. K. (2015) ,The electronic, magnetic and optical properties of double perovskites $\mathrm{A}_{2} \mathrm{FeReO}_{6}(\mathrm{~A}=\mathrm{Sr}, \mathrm{Ba})$ from first principles approach. Comp. Mat. Sci. 101, 313-320. 
Slater, J. C. (1960), Quantum Theory of Atomic Structure. McGraw-Hill, New York, Vol. 2, 310.

Smirnov, V. I. and Usvyat, D. E. (2001), Variational method for the generation of localized Wannier functions on the basis of Bloch functions. Phys. Rev. B 64, 245108.

Subramanian, M.A. (1988), Superconducting and magnetic behavior in $\mathrm{La}_{2-\mathrm{x}} \mathrm{Na}_{\mathrm{x}} \mathrm{CuO}_{4}$. Science 240, 495-497.

Vaughey, J. T., Wiley, J. B. and Poeppelmeier, K. R. (1991), Synthesis, structure, and properties of $\mathrm{LaSrCuGaO}_{5}$. Z. anorg. allg. Chem., 598: 327-338.

Wannier, G.H. (1937), The structure of electronic excitation levels in insulating crystals. Phys. Rev. 52, 191.
Wei Ku., Rosner, H., Pickett, W.E. and Scaletta, R.T. (2002), Insulating Ferromagnetism in $\mathrm{La}_{4} \mathrm{Ba}_{2} \mathrm{Cu}_{2} \mathrm{O}_{10}$ : An Ab InitioWannier Function Analysis, Phys. Rev. Lett. 89, 167204.

Yahua, Y., Feng, H., Ghimire, M. P., Matshushita,Y., Tsujimoto, Y., J. He, M. Tanaka, M., Katsuya, Y., and Yamaura, K. (2015), High-pressure synthesis, crystal structure, and magnetic properties of $5 \mathrm{~d}$ double perovskite oxides $\mathrm{Ca}_{2} \mathrm{MgOsO}_{6}$ and $\mathrm{Sr}_{2} \mathrm{MgOsO}_{6}$. Inorg. Chem. 54, 3422-3544.

$\mathrm{Yu}, \mathrm{J}$, Freeman, A. J. and $\mathrm{Xu}$, J. -H (1987), Electronically driven instabilities and superconductivity in the layered $\mathrm{La}_{2-\mathrm{x}} \mathrm{Ba}_{\mathrm{x}} \mathrm{CuO}_{4}$ perovskites. Phys. Rev. Lett. 58, 1035-1036. 